\title{
Six Biophysical Screening Methods Miss a Large Proportion of Crystallographically Discovered Fragment Hits: A Case Study
}

Johannes Schiebel ${ }^{1}$, Nedyalka Radeva ${ }^{1}$, Stefan G. Krimmer ${ }^{1}$, Xiaojie Wang ${ }^{1}$, Martin Stieler ${ }^{1}$, Frederik R. Ehrmann ${ }^{1}$, Kan Fu ${ }^{1}$, Alexander Metz ${ }^{1}$, Franziska U. Huschmann ${ }^{1,2}$, Manfred S. Weiss ${ }^{2}$, Uwe Mueller ${ }^{2}$, Andreas Heine ${ }^{1} \&$ Gerhard Klebe $^{1 *}$

1) Institut für Pharmazeutische Chemie, Philipps-Universität Marburg Marbacher Weg 6, 35032 Marburg (Germany)

2) Helmholtz-Zentrum Berlin für Materialien und Energie, HZB, BESSY II, Abteilung Makromolekulare Kristallographie

Albert-Einstein-Str. 15, 12489 Berlin (Germany)

*) E-mail: klebe@mailer.uni-marburg.de 


\section{Table of Contents}

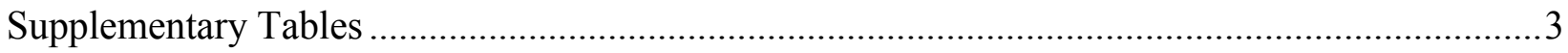

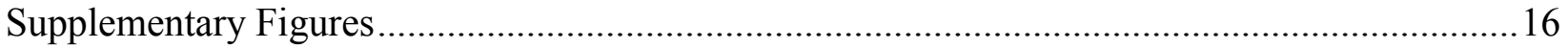

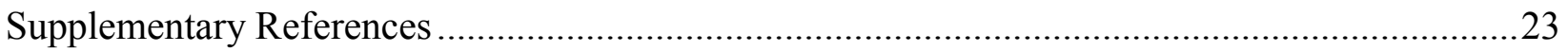




\section{Supplementary Tables}

Table S1. Method-specific hits never confirmed by any other technique

\begin{tabular}{|c|c|}
\hline Screening method & $\begin{array}{c}\text { No. and fraction of hits identified by } \\
\text { only this single biophysical method }\end{array}$ \\
\hline X-ray crystallography & 31 out of 71 X-ray hits $=44 \%$ \\
HCS & 18 out of 56 HCS hits $=32 \%$ \\
RDA & 11 out of 50 RDA hits $=22 \%$ \\
NMR & 5 out of 22 NMR hits $=23 \%$ \\
ESI-MS & 2 out of 8 ESI-MS hits $=25 \%$ \\
TSA & 5 out of 25 TSA hits $=20 \%$ \\
MST & 16 out of 36 MST hits $=44 \%$ \\
\hline
\end{tabular}


Table S2. Comparison of the performance of each applied screening method (see also Figures 4 and S2) ${ }^{a}$

\begin{tabular}{|c|c|c|c|c|c|c|c|}
\hline \multirow[t]{2}{*}{ Screening method } & \multirow[t]{2}{*}{$\begin{array}{c}A U C \\
(\boldsymbol{R O C})^{b}\end{array}$} & \multicolumn{2}{|c|}{$\begin{array}{l}\text { Fraction of the } \\
\text { library to be } \\
\text { screened / } \%^{c}\end{array}$} & \multicolumn{2}{|c|}{$\begin{array}{l}\text { Fraction of all } \\
\text { structures } \\
\text { retrieved / \% }\end{array}$} & \multicolumn{2}{|c|}{$\begin{array}{l}\text { Crystallographic } \\
\text { hit rate / } \%\end{array}$} \\
\hline & & $\begin{array}{l}\text { chosen } \\
\text { cutoff }^{f}\end{array}$ & $\begin{array}{l}\text { optimal } \\
\text { cutoff }^{g}\end{array}$ & $\begin{array}{l}\text { chosen } \\
\text { cutoff }^{f}\end{array}$ & $\begin{array}{l}\text { optimal } \\
\text { cutoff }\end{array}$ & $\begin{array}{l}\text { chosen } \\
\text { cutoff }\end{array}$ & $\begin{array}{l}\text { optimal } \\
\text { cutoff }\end{array}$ \\
\hline HCS & 0.63 & 17.2 & 28.9 & 34.4 & 47.5 & 37.5 & 30.9 \\
\hline RDA & 0.70 & 14.0 & 43.0 & 39.7 & 70.6 & 54.0 & 31.2 \\
\hline NMR (binding) & 0.57 & 34.5 & 59.7 & 40.4 & 68.1 & 26.8 & 26.0 \\
\hline NMR (competition) & 0.60 & 14.6 & 60.7 & 29.8 & 68.1 & 46.7 & 25.6 \\
\hline ESI-MS & 0.49 & 2.3 & 2.3 & 6.1 & 6.1 & 50.0 & 50.0 \\
\hline TSA & 0.61 & 7.6 & 12.4 & 17.5 & 31.7 & 44.0 & 48.8 \\
\hline MST & 0.47 & 12.8 & 10.6 & 16.9 & 16.9 & 27.8 & 33.3 \\
\hline
\end{tabular}

${ }^{a}$ All values refer to those fragments that could be successfully analyzed by each respective method ${ }^{1}$

${ }^{b}$ The area under the curve (AUC) of the respective receiver operator characteristic (ROC) curve was chosen as performance measure. Please note that the ROC plots will slightly differ from the enrichment plots shown in Figures 4 and S2 because they plot the true positive (fraction of structures) against the false positive rate and not against the investigated fraction of the library. Thus, the ideal ROC curve would be a vertical line at a false positive rate of $0 \%$.

${ }^{c}$ Percentage of the library suggested as putative hits at a given hit-definition cutoff. This fraction would subsequently be screened by crystallography using the respective biophysical method as pre-filter.

${ }^{d}$ Percentage of all structures predicted by the respective method at a given cutoff.

${ }^{e}$ Resulting X-ray hit rate when crystallographically investigating only the hits suggested by the respective method at a given cutoff.

${ }^{f}$ Hit-definition threshold chosen based on previous experience (same color as in Figures 4 and S2, which also contain the explicit threshold values). ${ }^{1}$

${ }^{g}$ Hit-definition threshold up to which the respective method maintains an above-random performance (same color as in Figures 4 and S2, which also contain the explicit threshold values). 
Table S3. Data collection and (automatic) refinement statistics for the time-dependent soaking experiments with fragment $112{ }^{a}$

\begin{tabular}{|c|c|c|c|c|c|c|}
\hline & $2 \min$ & $10 \mathrm{~min}$ & $20 \min$ & $2 \mathrm{~h}$ & $20 \mathrm{~h}$ & $48 h^{b}$ \\
\hline Data collection & & & & & & \\
\hline Wavelength $(\AA)$ & 0.894 & 0.894 & 0.894 & 0.918 & 0.918 & 0.895 \\
\hline Cell dimensions & & & & & & \\
\hline a, b, c $(\AA)$ & $45.2,72.7,52.5$ & $45.3,72.8,52.6$ & $45.3,72.9,52.7$ & $45.3,73.5,52.7$ & $45.5,73.2,52.9$ & $45.4,72.8,52.8$ \\
\hline$\alpha, \beta, \gamma\left({ }^{\circ}\right)$ & $90,109.4,90$ & $90,109.5,90$ & $90,109.6,90$ & $90,109.7,90$ & $90,109.7,90$ & $90,109.6,90$ \\
\hline Space group & & & & & & \\
\hline Resolution $(\AA)^{c}$ & $\begin{array}{l}42.7-1.15 \\
(1.22-1.15)\end{array}$ & $\begin{array}{l}42.7-1.33 \\
(1.41-1.33)\end{array}$ & $\begin{array}{l}42.7-1.20 \\
(1.27-1.20)\end{array}$ & $\begin{array}{l}42.7-1.35 \\
(1.43-1.35)\end{array}$ & $\begin{array}{l}42.9-1.36 \\
(1.44-1.36)\end{array}$ & $\begin{array}{l}42.7-1.40 \\
(1.49-1.40)\end{array}$ \\
\hline Unique reflections & $113,274(18,244)$ & $73,837(11,886)$ & $98,030(15,450)$ & $71,205(11,441)$ & $70,025(11,055)$ & $62,976(10,055)$ \\
\hline Completeness (\%) & 99.7 (99.7) & 99.8 (99.7) & $97.2(95.3)$ & $99.7(99.8)$ & $99.3(97.5)$ & $99.6(98.6)$ \\
\hline Average redundancy & $4.2(4.0)$ & $4.2(4.1)$ & $4.3(4.3)$ & $4.2(4.1)$ & $4.1(4.0)$ & $4.2(4.1)$ \\
\hline $\mathrm{R}_{\text {merge }}(\%)$ & $5.3(46.3)$ & $5.6(49.9)$ & $5.3(47.6)$ & $6.4(50.5)$ & $6.9(49.6)$ & $4.3(39.6)$ \\
\hline$\langle\mathrm{I} / \sigma(\mathrm{I})\rangle$ & $16.0(2.9)$ & $18.4(2.9)$ & $17.4(3.1)$ & $15.9(3.1)$ & $13.0(2.8)$ & $22.5(3.8)$ \\
\hline Wilson B factor $\left(\AA^{2}\right)$ & 8.8 & 9.5 & 8.7 & 9.1 & 10.6 & 10.7 \\
\hline Refinement & & & & & & \\
\hline Resolution $(\AA ̊)$ & $28.0-1.15$ & $28.1-1.33$ & $42.7-1.20$ & $28.0-1.35$ & $28.1-1.36$ & $29.4-1.40$ \\
\hline $\mathrm{R}_{\text {cryst }}(\%)$ & 13.2 & 12.9 & 12.6 & 13.3 & 14.4 & 13.5 \\
\hline $\mathrm{R}_{\text {free }}(\%)$ & 14.9 & 14.9 & 13.9 & 15.8 & 16.9 & 17.0 \\
\hline rmsd bond lengths $(\AA)$ & 0.006 & 0.007 & 0.007 & 0.007 & 0.007 & 0.007 \\
\hline rmsd bond angles $\left({ }^{\circ}\right)$ & 1.21 & 1.19 & 1.22 & 1.21 & 1.18 & 1.18 \\
\hline $\begin{array}{l}\text { Average B-factor for } \\
\text { protein }\left(\AA^{2}\right)\end{array}$ & 10.3 & 10.6 & 9.9 & 9.8 & 11.7 & 11.3 \\
\hline
\end{tabular}

${ }^{a}$ All soaking experiments were conducted for the given time spans at a fragment concentration of $90 \mathrm{mM}$

${ }^{b}$ Equal to the experiment at $90 \mathrm{mM}$ fragment concentration (Table S4, column 6)

${ }^{c}$ Values in parenthesis refer to the highest resolution shell 
Table S4. Data collection and (automatic) refinement statistics for the concentration-dependent soaking experiments with fragment $112^{a}$

\begin{tabular}{|c|c|c|c|c|c|}
\hline & $90 \mu \mathrm{M}$ & $900 \mu \mathrm{M}$ & $4.5 \mathrm{mM}$ & $9 \mathrm{mM}$ & $90 \mathbf{~ m M}^{b}$ \\
\hline $\begin{array}{l}\text { Data collection } \\
\text { Wavelength }(\AA)\end{array}$ & 0918 & 0918 & 0894 & 0918 & 0895 \\
\hline Cell dimensions & & & & & \\
\hline a, b, c $(\AA)$ & $45.4,73.1,52.8$ & $45.4,73.2,52.9$ & $45.2,73.0,52.6$ & $45.2,73.3,52.6$ & $45.4,73.1,52.8$ \\
\hline$\alpha, \beta, \gamma\left({ }^{\circ}\right)$ & $90,109.6,90$ & $90,109.6,90$ & $90,109.3,90$ & $90,109.5,90$ & $90,109.6,90$ \\
\hline Space group & & & & & $\mathrm{P} 2_{1}$ \\
\hline Resolution $(\AA)^{c}$ & $\begin{array}{l}42.7-1.15 \\
(1.22-1.15)\end{array}$ & $\begin{array}{l}42.8-1.50 \\
(1.59-1.50)\end{array}$ & $\begin{array}{l}42.7-1.25 \\
(1.33-1.25)\end{array}$ & $\begin{array}{l}42.6-1.42 \\
(1.51-1.42)\end{array}$ & $\begin{array}{l}42.7-1.40 \\
(1.49-1.40)\end{array}$ \\
\hline Unique reflections & $113,994(17,772)$ & $52,144(8,412)$ & $87,097(13,770)$ & $60,702(9,656)$ & $62,976(10,055)$ \\
\hline Completeness (\%) & $99.1(95.9)$ & $99.8(99.8)$ & $97.7(96.0)$ & $99.6(98.2)$ & $99.6(98.6)$ \\
\hline Average redundancy & $4.0(3.4)$ & $4.2(4.2)$ & $4.3(4.2)$ & $4.1(3.7)$ & $4.2(4.1)$ \\
\hline $\mathrm{R}_{\text {merge }}(\%)$ & $5.1(49.1)$ & $8.0(48.7)$ & $5.2(46.9)$ & $6.9(50.7)$ & $4.3(39.6)$ \\
\hline$\langle\mathrm{I} / \sigma(\mathrm{I})\rangle$ & $15.8(2.5)$ & $14.5(3.5)$ & $18.0(3.4)$ & $13.7(2.8)$ & $22.5(3.8)$ \\
\hline Wilson B factor $\left(\AA^{2}\right)$ & 9.4 & 10.0 & 9.1 & 11.4 & 10.7 \\
\hline Refinement & & & & & \\
\hline Resolution $(\AA)$ & $28.1-1.15$ & $39.7-1.50$ & $28.1-1.25$ & $28.0-1.42$ & 29.4-1.40 \\
\hline $\mathrm{R}_{\text {cryst }}(\%)$ & 13.5 & 14.2 & 12.6 & 13.5 & 13.5 \\
\hline $\mathrm{R}_{\text {free }}(\%)$ & 14.9 & 17.8 & 14.5 & 17.0 & 17.0 \\
\hline rmsd bond lengths $(\AA)$ & 0.007 & 0.008 & 0.007 & 0.007 & 0.007 \\
\hline rmsd bond angles $\left({ }^{\circ}\right)$ & 1.23 & 1.20 & 1.22 & 1.19 & 1.18 \\
\hline $\begin{array}{l}\text { Average B-factor for } \\
\text { protein }\left(\AA^{2}\right)\end{array}$ & 10.7 & 10.5 & 10.6 & 12.3 & 11.3 \\
\hline
\end{tabular}

${ }^{a}$ All soaking experiments were conducted for $48 \mathrm{~h}$ at the given fragment concentrations

${ }^{b}$ Equal to the $48 \mathrm{~h}$ soaking experiment (Table S3, column 7)

${ }^{c}$ Values in parenthesis refer to the highest resolution shell 
Table S5. Binding affinity data obtained by isothermal titration calorimetry (ITC) for all 71 X-ray hits

\begin{tabular}{|c|c|c|c|c|c|}
\hline ID & Structure $^{a}$ & $\Delta \mathbf{G}^{\circ}\left(\mathrm{kJ} \mathrm{mol}^{-1}\right)$ & $K_{d}(\mathbf{m M})$ & 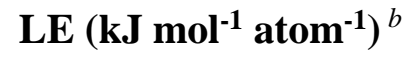 & $\operatorname{Assays}^{c}$ \\
\hline 4 & & $>-11.4$ & $>10.0$ & $\mathrm{NA}^{d}$ & 0 \\
\hline 5 & & $-15.9 \pm 0.5$ & 1.7 & 1.59 & 3 \\
\hline 14 & & $-13.1 \pm 0.4$ & 5.0 & 1.01 & 1 \\
\hline 17 & & $>-11.4$ & $>10.0$ & $\mathrm{NA}^{d}$ & 4 \\
\hline 31 & & $-13.0 \pm 0.9$ & 5.2 & 1.00 & 0 \\
\hline 34 & & $\mathrm{NA}^{e}$ & $\mathrm{NA}^{e}$ & $\mathrm{NA}^{e}$ & 1 \\
\hline 35 & & $-14.2 \pm 0.1$ & 3.2 & 1.18 & 0 \\
\hline 39 & & $-14.6 \pm 0.9$ & 2.8 & 0.91 & 0 \\
\hline
\end{tabular}




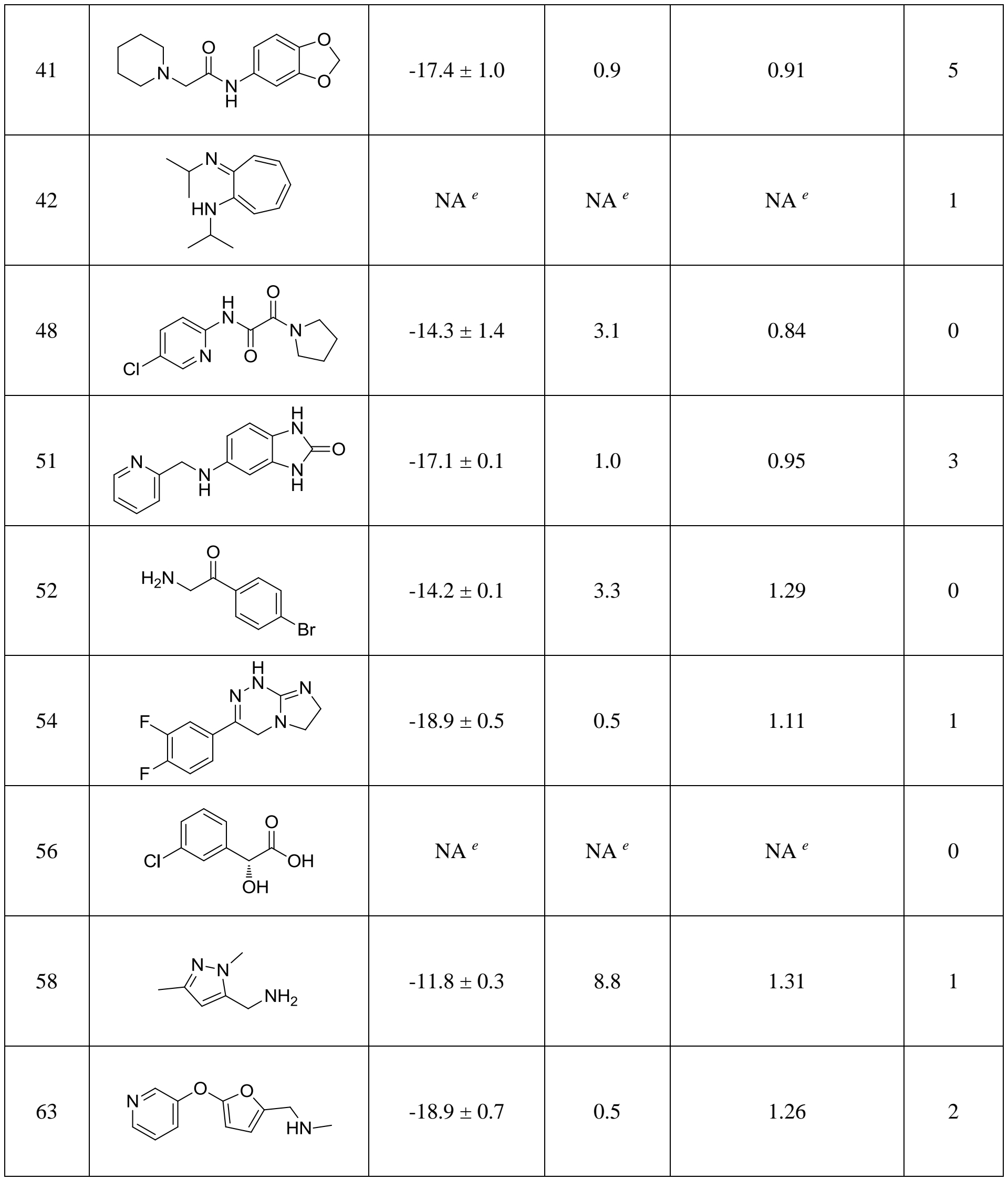




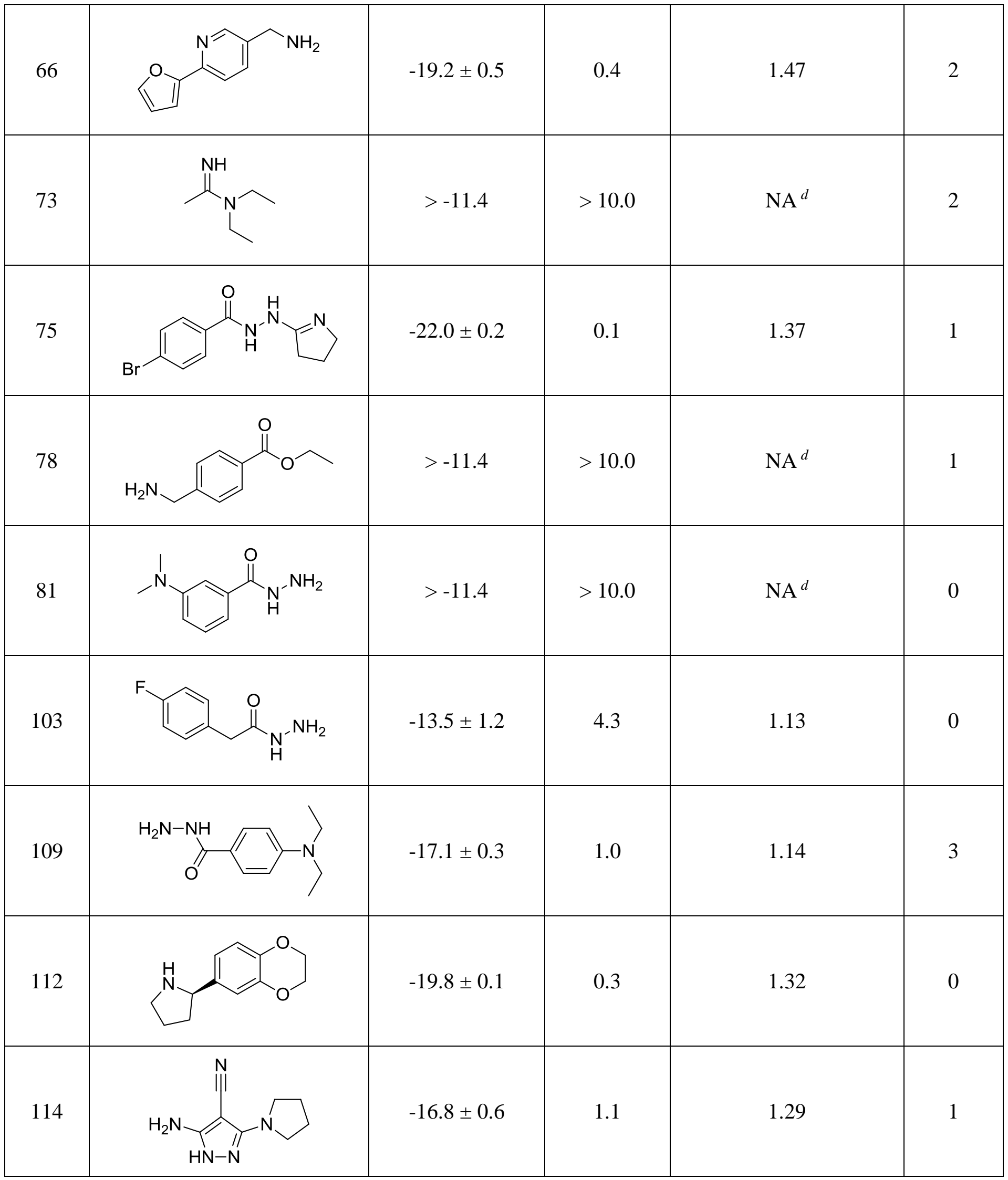




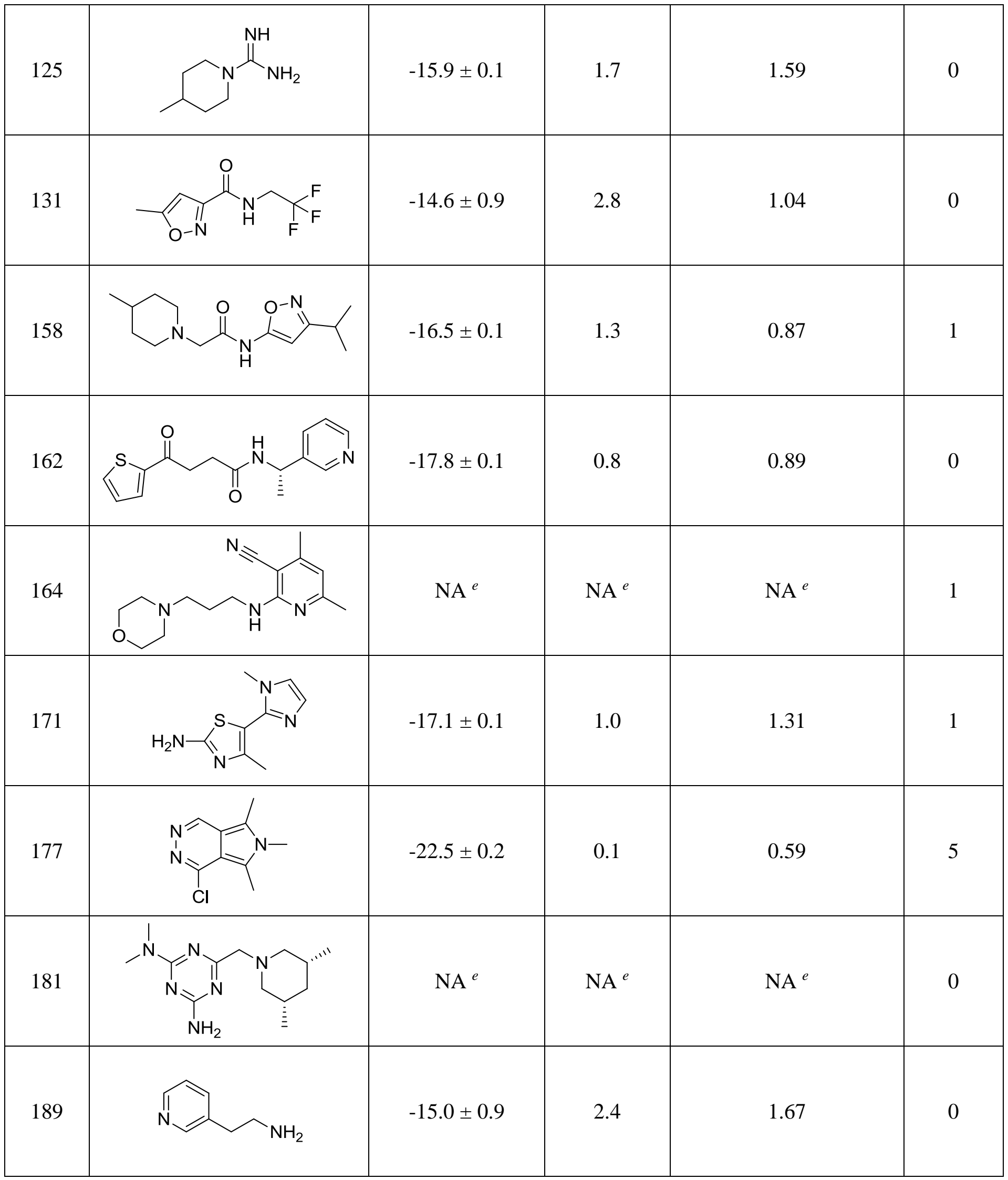




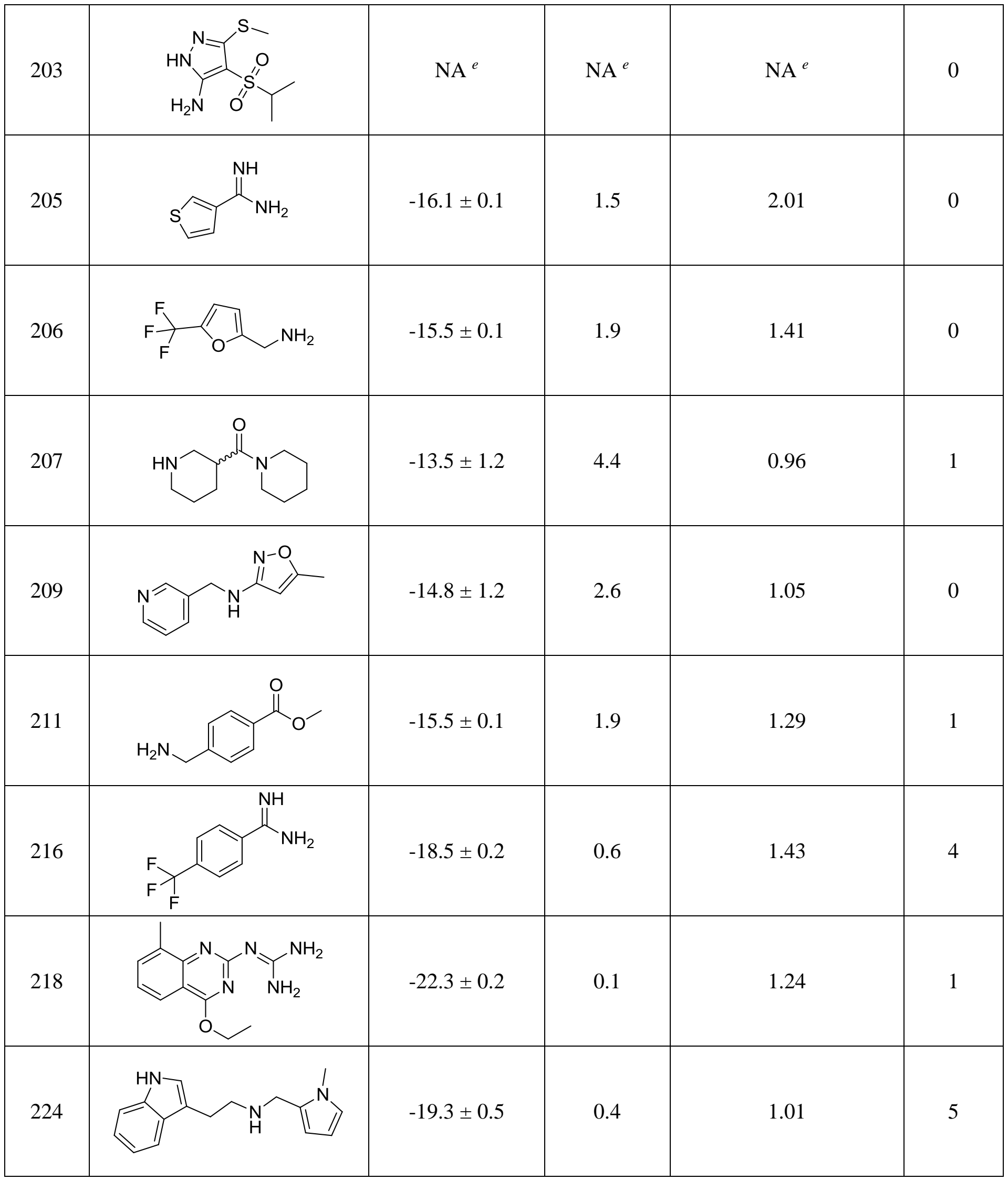




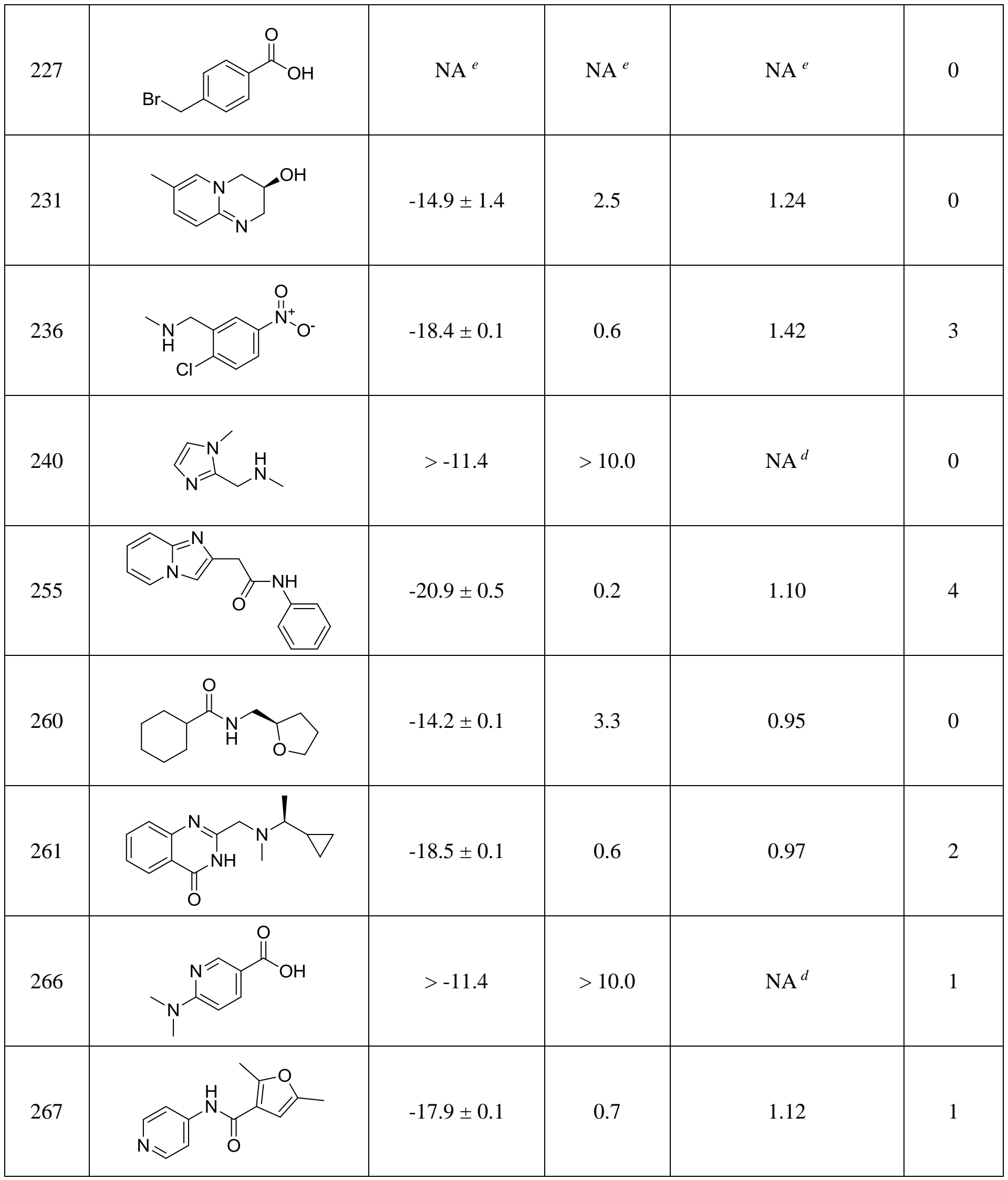




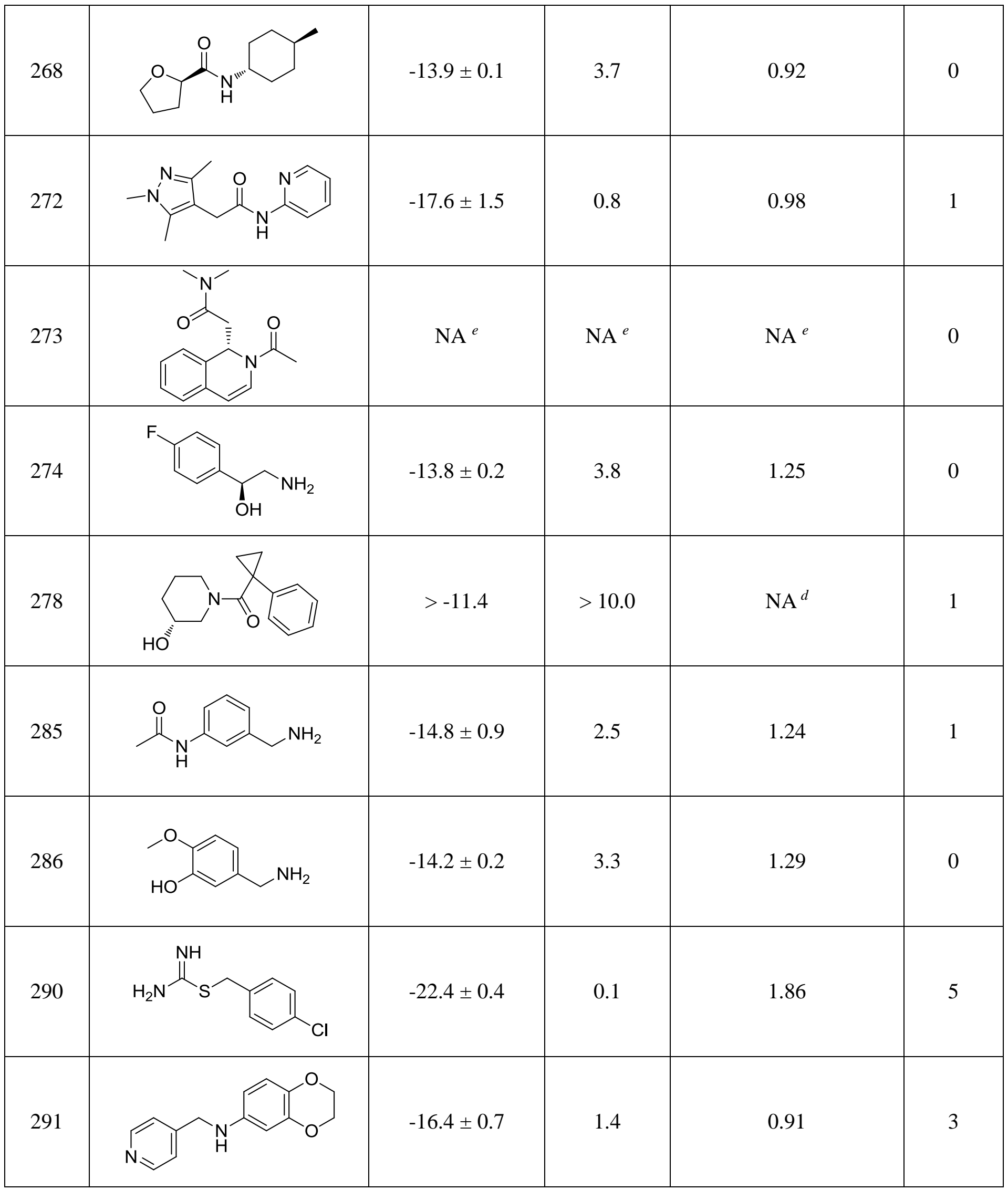




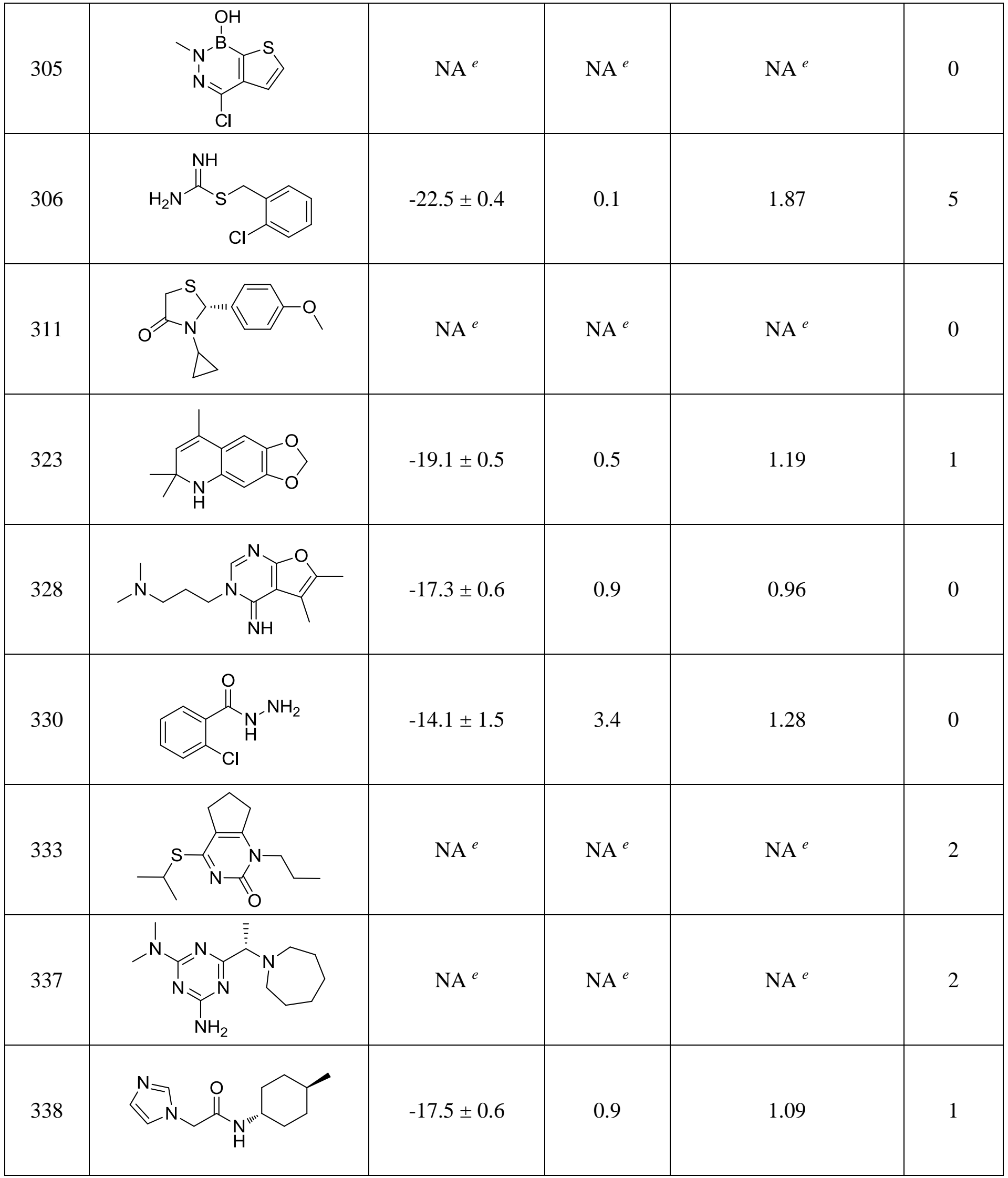

${ }^{a}$ Fragments were purchased and used as racemic mixtures in all experiments. Depicted is the stereoisomer observed in the crystal structure (in case of fragment 207 both stereoisomers bound to EP at different sites). 
${ }^{b}$ The ligand efficiency (LE) was calculated as $-\Delta \mathrm{G}^{\circ}$ divided by the number of non-hydrogen atoms (in $\mathrm{kJ} \mathrm{mol}^{-1}$ atom ${ }^{-1}$ ).

${ }^{c}$ This number indicates how many screening assays identified the respective fragment as hit.

${ }^{d}$ No exact LE could be calculated for these weak-binding fragments since the binding free energy had an experimental error beyond the designated limit.

${ }^{e}$ No ITC data available for these compounds because the displacement ligand SAP114 (Figure S4) cannot expel the bound fragment due to non-overlapping binding positions. 


\section{Supplementary Figures}

a)
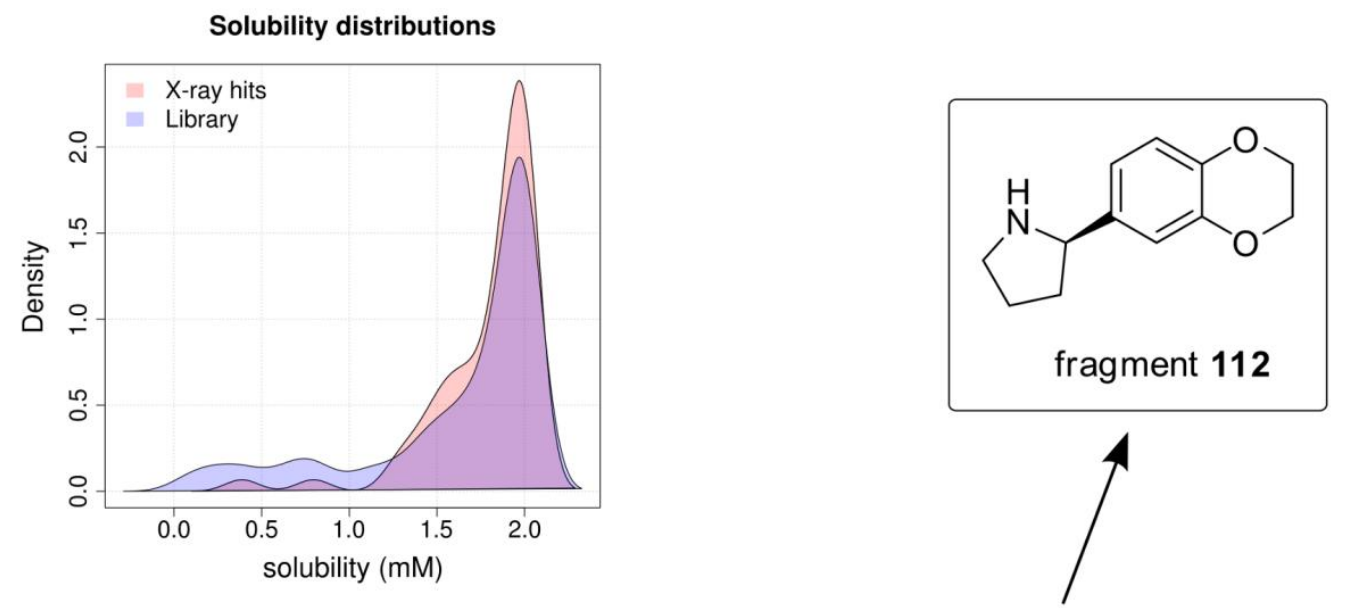

b)
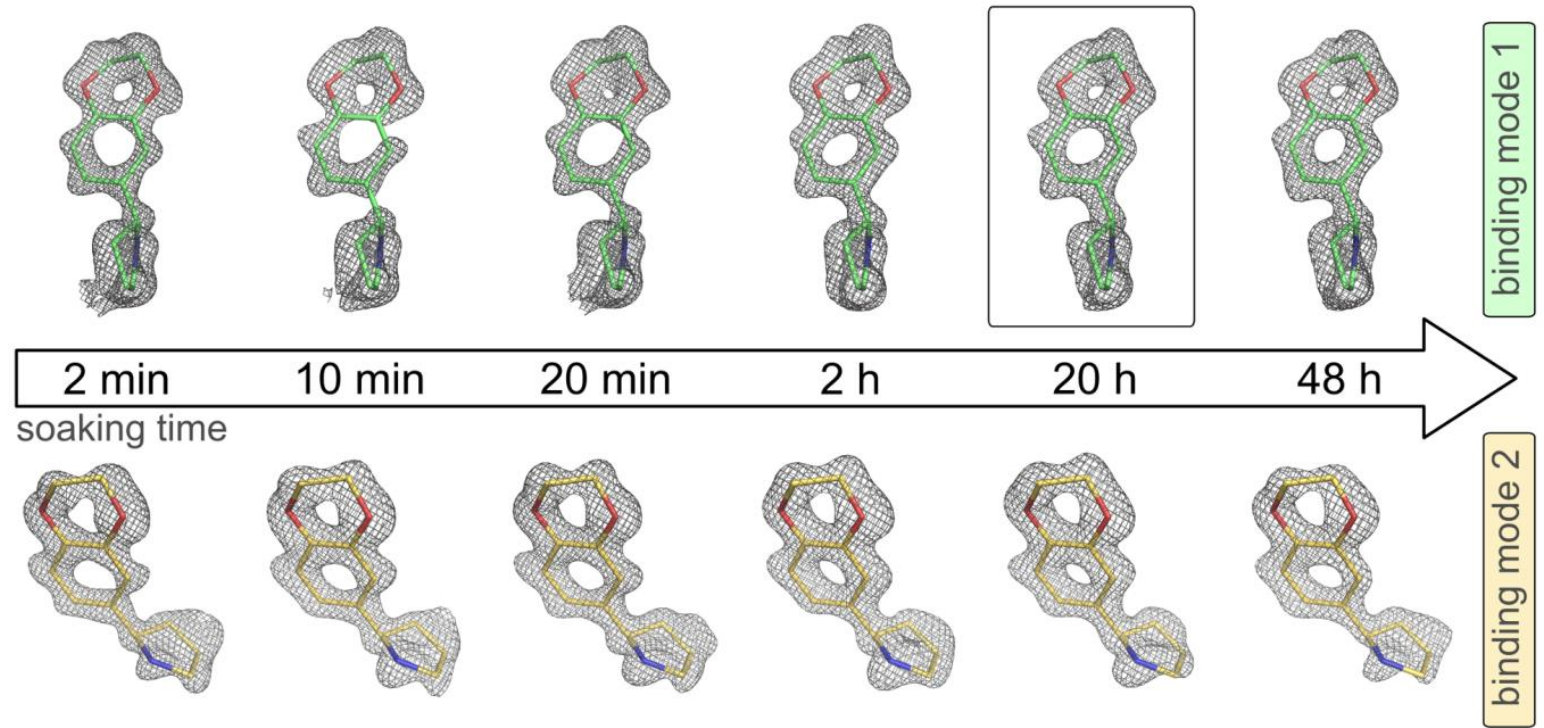

c)
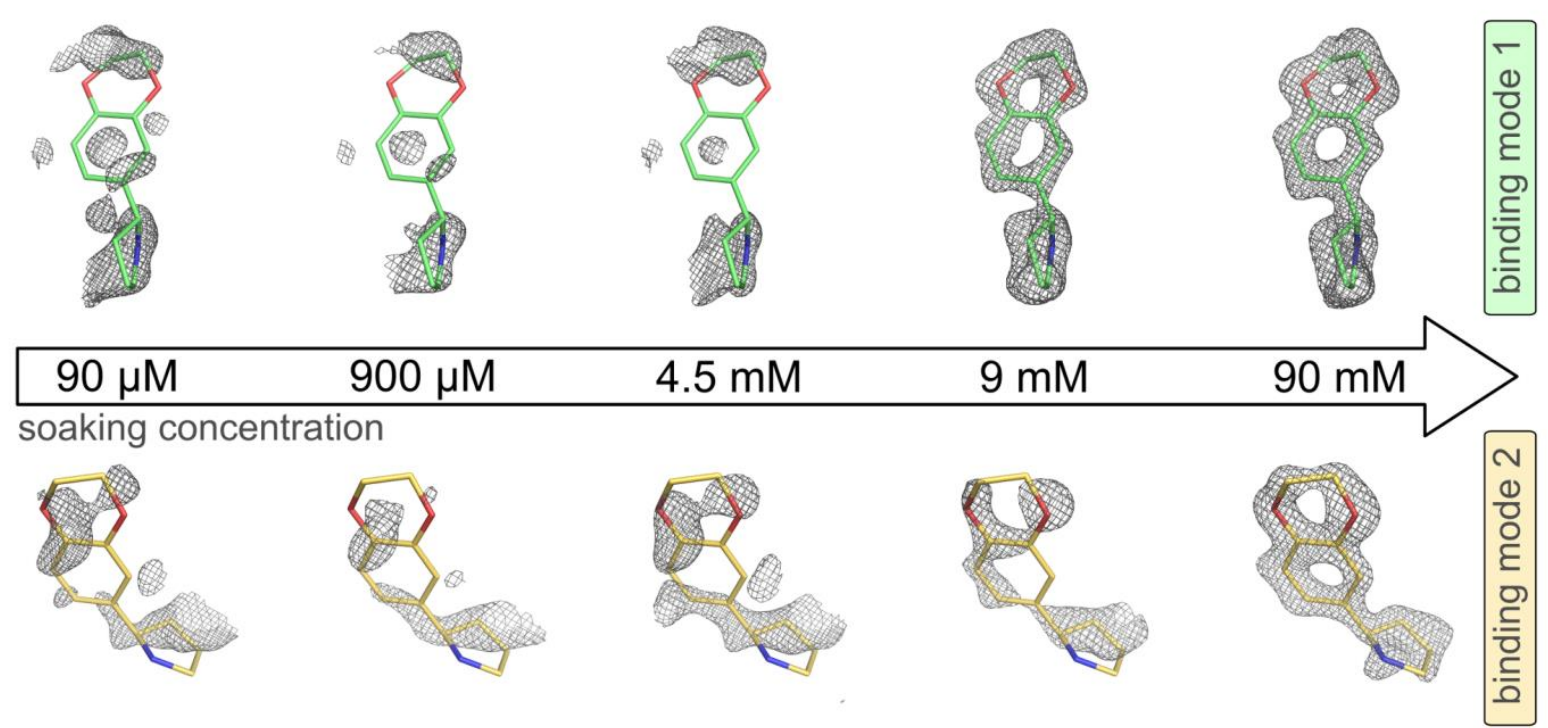
Figure S1. Influence of solubility, soaking time and concentration on hit identification. a) Comparison of the experimental solubility ${ }^{1,2}$ between X-ray binders and all fragments in the library. The plot compares the solubility kernel density estimations of all 324 fragments (blue) for which the solubility could be determined with those identified as X-ray hits (pink). All solubility values $\geq 2 \mathrm{mM}$ were summarized in the $2 \mathrm{mM}$ bin. The depicted graph was generated using the statistical framework R. b-c) Influence of soaking time (b) and concentration (c) on the crystallographic detection of fragment 112. While the time-dependent soaking experiments were conducted at $90 \mathrm{mM}$ fragment concentration, the concentration-dependent experiments were performed using a soaking time of $48 \mathrm{~h}$. The two bound copies of fragment 112 located at different sites of the EP pocket are depicted as green and yellow sticks. All structures were solved applying exactly the same procedure using an in-house automatic refinement pipeline (for details see Supplementary Experimental Section). Respective $\mathrm{mF}_{\mathrm{o}}-\mathrm{DF}_{\mathrm{c}}$ maps are shown prior to the inclusion of any fragment at the $3 \sigma$ level as gray meshes. 
a)

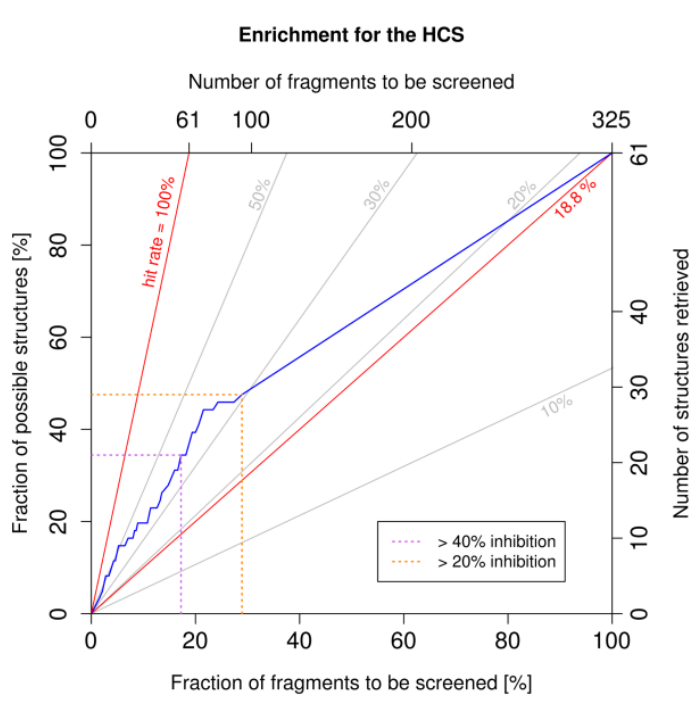

c)

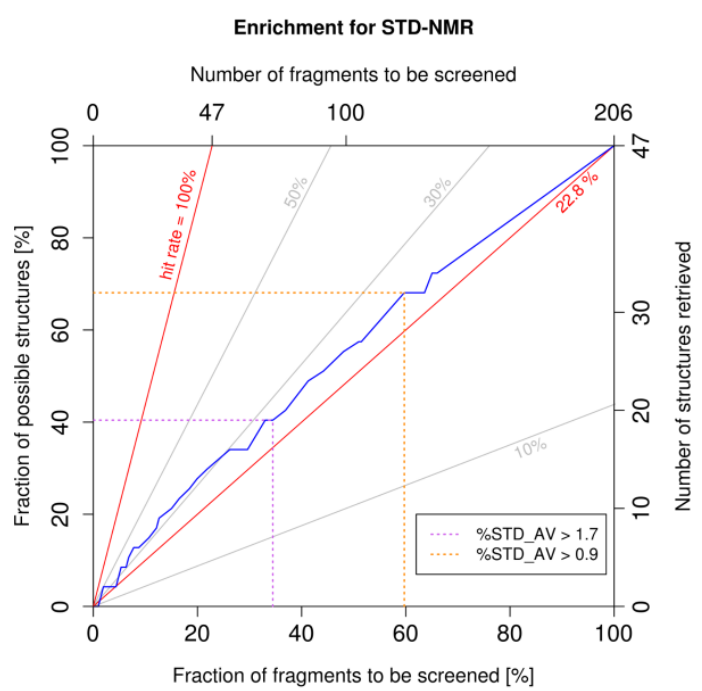

e)

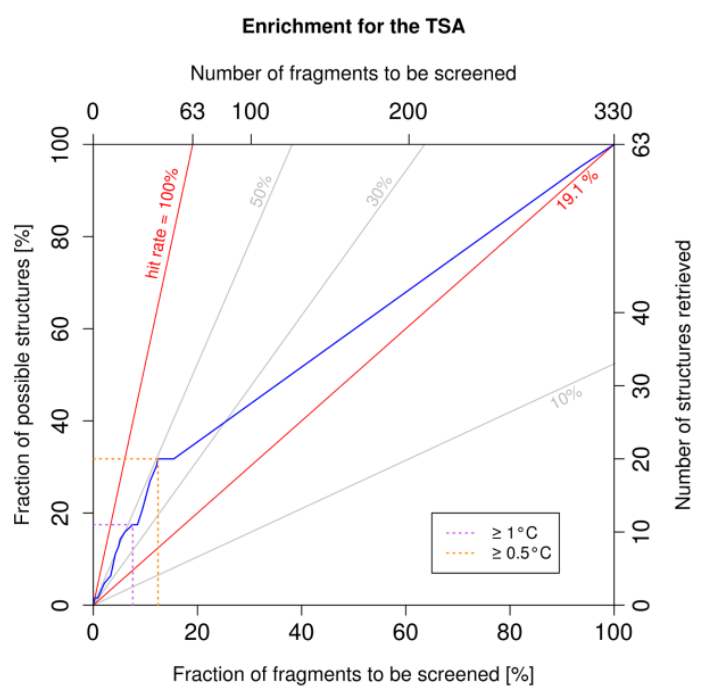

b)

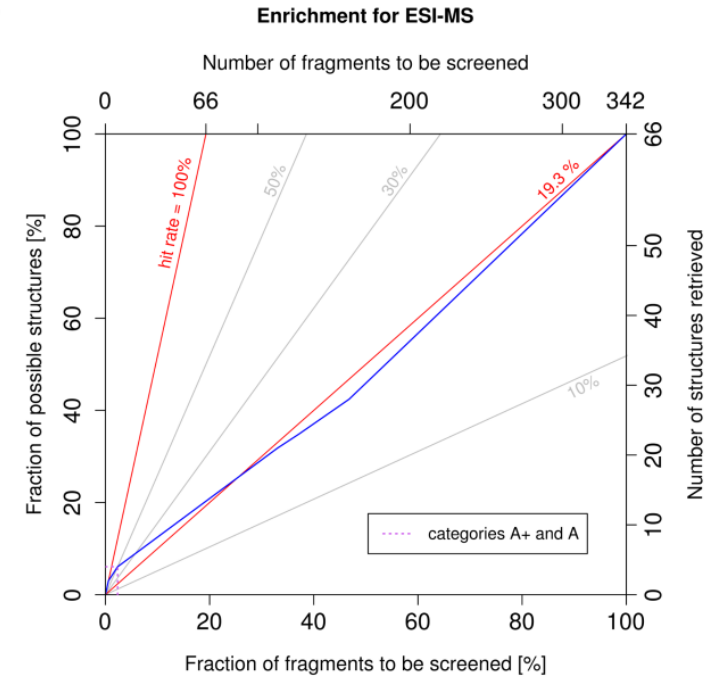

d) Enrichment for STD-NMR (competition experiment)

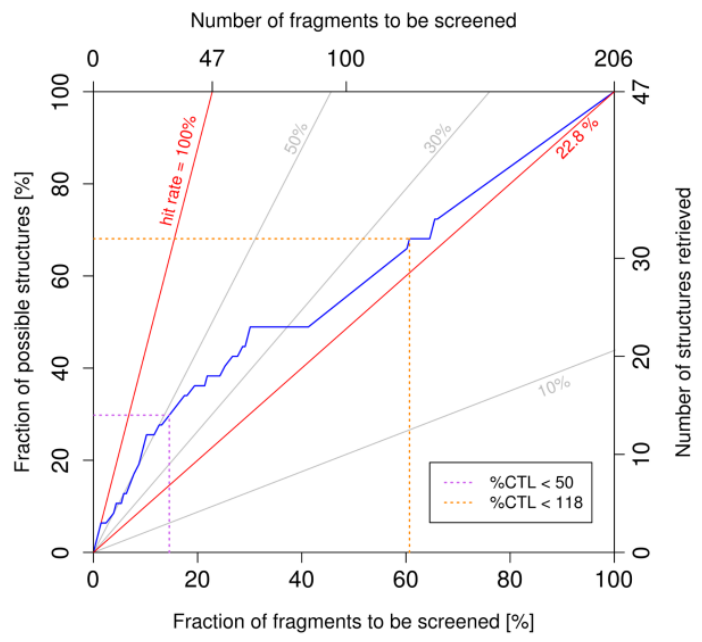

f)

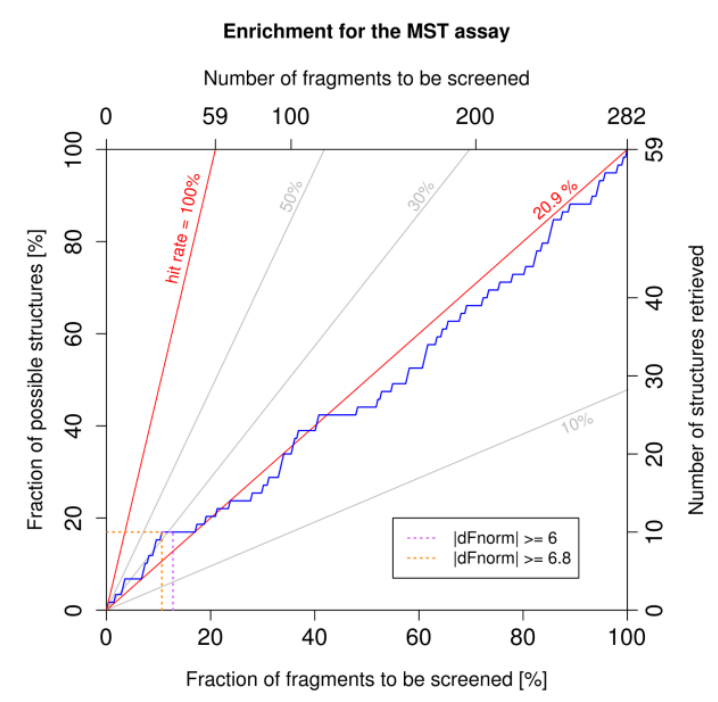


Figure S2. Predictive power of different screening methods with respect to the identification of crystallographic hits. For these enrichment analyses, the list of those fragments that could be investigated by each technique was sorted by decreasing score (for details see below). Each list was then worked through from top-to-bottom and the number and percentage of fragments that would have been analyzed in subsequent crystallographic experiments based on a certain hitdefinition cutoff was plotted against the number and percentage of structures retrieved from these trials, resulting in the blue enrichment curves. The hit-definition thresholds applied in this study and chosen based on previous experience are indicated by the purple dotted lines, ${ }^{1}$ while the optimal cutoffs up to which each method maintains an above-random performance are highlighted in orange (omitted for ESI-MS since our cutoff choice was already optimal). The resulting characteristics at these cutoffs are given in Table S2. Crystallographic hit rates at a certain hit-definition cutoff can be estimated from the intersection of the blue and gray lines (e.g. the hit rate for the HCS at the $40 \%$ inhibition cutoff is $38 \%$ ). The two red lines indicate how the blue curve would look like for an optimal or random selection of fragments, respectively. These plots were generated using the statistical framework R. a) Enrichment for the HCS. The percentage of inhibition was used as score. Since the assay was not sensitive enough to distinguish between fragments with inhibition levels below $20 \%, 1,2$ the right part of the blue curve is linear. b) Enrichment for the ESI-MS screen. Each fragment investigated by MS was categorized into one of six groups denoted as $\mathrm{A}+, \mathrm{A}, \mathrm{B}, \mathrm{WB} *$, NS and WB (from most to least interesting; for details please refer to reference ${ }^{1}$ ). These categories were used as a score and explain the step-function like appearance of the enrichment curve. In particular, it can be clearly seen that the first two categories comprising only 8 fragments lead to an enrichment while the further classes do not discriminate well between crystallographic binders and non-binders. c-d) Enrichment for the STD-NMR screen. Here, results from the primary binding experiment (c) were confirmed by a secondary competition experiment using ritonavir as displacement ligand 
(d). ${ }^{1}$ Since for 70 fragments the signal to noise ratio in the STD spectra was too low for integration (indicating no binding), ${ }^{1}$ the right parts of the blue curves are linear. e) Enrichment for the TSA. For this screen, the score is represented by the observed thermal stabilization of EP upon binding of the respective fragment $\left(\Delta \mathrm{T}_{\mathrm{m}}\right)$. The TSA was performed in two steps. ${ }^{1}$ In a primary screen, samples were heated using an increment of $0.5^{\circ} \mathrm{C}$ per minute. All fragments that stabilized EP by more than $0.8^{\circ} \mathrm{C}$, corresponding to the 2-fold standard deviation for measurements of the apo-protein, were then re-evaluated in duplicate or triplicate using a finer increment of $0.2^{\circ} \mathrm{C}$ per minute. Those 21 fragments that had a $\Delta \mathrm{T}_{\mathrm{m}}<-0.8^{\circ} \mathrm{C}$ in the primary screen were ranked worst followed by those 258 fragments that displayed a $\Delta \mathrm{T}_{\mathrm{m}}$ between -0.8 and $+0.8^{\circ} \mathrm{C}$. The remaining 51 fragments that were also investigated in the secondary screen were sorted by decreasing $\Delta \mathrm{T}_{\mathrm{m}}$ values at the top of the list. f) Enrichment for the MST screen (performed at a fragment concentration of $0.5 \mathrm{mM}^{1}$ ). Here, the absolute differences between normalized fluorescence values of fragment-containing samples and fragment-free negative controls $\left(\left|\Delta \mathrm{F}_{\text {norm }}\right|\right)$ were used as scores. 

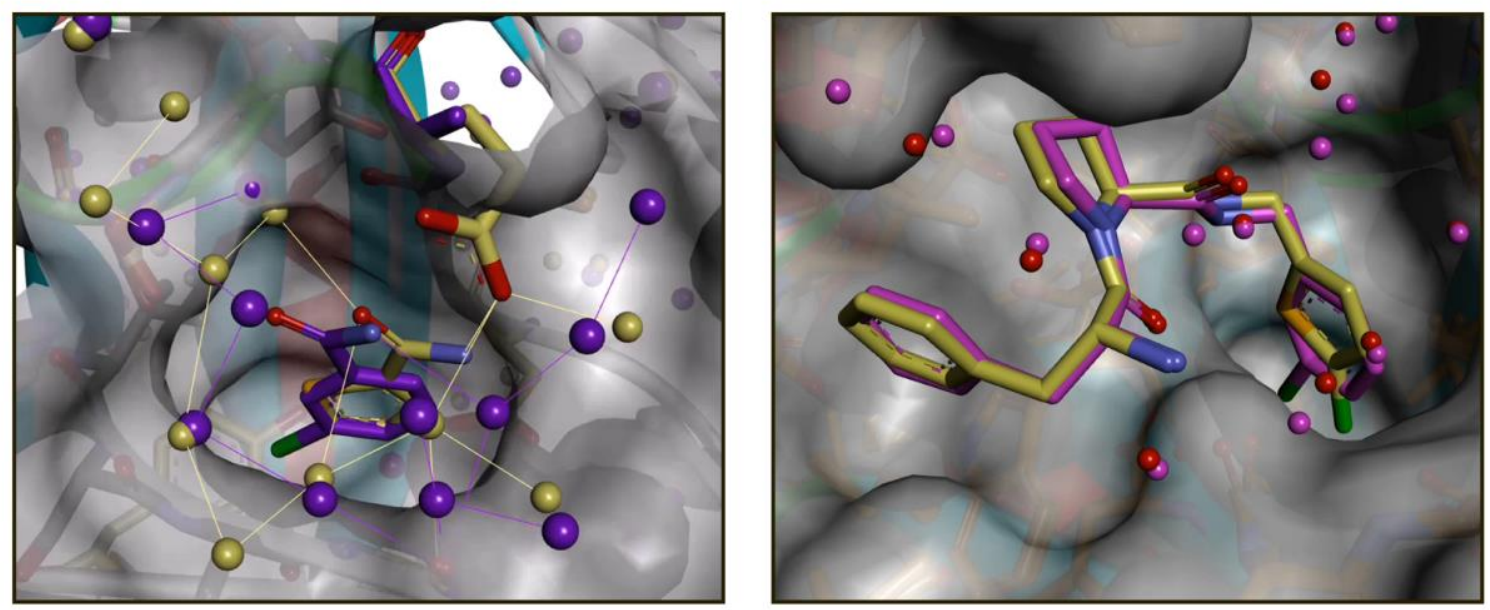

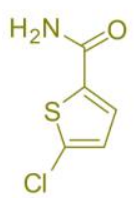

$\mathrm{K}_{\mathrm{i}}=430 \mu \mathrm{M}$

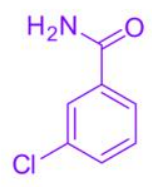

Optimization

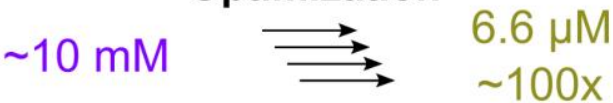

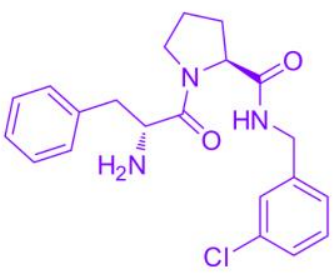

$180 \mathrm{nM}$ $\sim 55000 \mathrm{x}$

Figure S3. Two fragments, chloro-thiophene carboxamide and m-chlorophenyl carboxamide, bind with very similar binding modes to the serine protease thrombin (left). Their binding affinities $\left(\mathrm{K}_{\mathrm{i}}\right)$ differ strongly, as the thiophene derivative binds with $430 \mu \mathrm{M}$ affinity, whereas the phenyl analog achieves only millimolar binding. ${ }^{3}$ This affinity difference can be explained by the more perfect water network sealing the S1 pocket of the protease and by the interaction with Glu192 in the thiophene example, which are less well established or missing for the phenyl fragment. Optimization of the initial fragments to tripeptide-like D-Phe-Pro-XXX derivatives (right) resulted in a $\sim 100$-fold or 55,000-fold potency enhancement leading to the expanded thiophene derivative with $6.6 \mu \mathrm{M}$ binding affinity and to the m-chlorophenyl analog with an affinity of $180 \mathrm{nM} .{ }^{4,5}$ Here the affinity difference results from a better chloro-aromatic contact between the P1-chloro substituent and Tyr228 in the latter case. In consequence, the weaker binding fragment finally resulted in the more potent optimized tripeptidic inhibitor. The images have been produced with the Discovery Studio Visualizer 4.0, Accelrys Software Inc. 


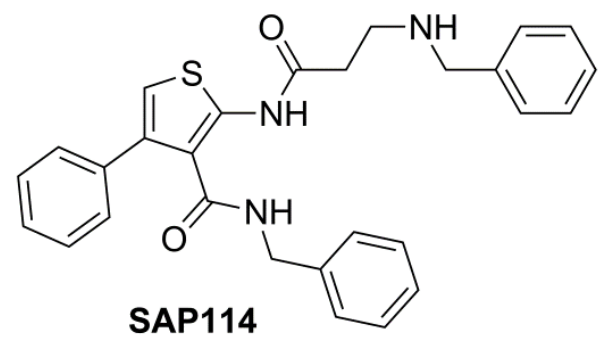

Figure S4. ITC displacement ligand. The chemical structure of SAP114 used for the ITC titrations is depicted (molecule 9 in reference ${ }^{6}$ ). 


\section{Supplementary References}

1. Schiebel, J., Radeva, N., Köster, H., Metz, A., Krotzky, T., Kuhnert, M., Diederich, W. E., Heine, A., Neumann, L., Atmanene, C., Roecklin, D., Vivat-Hannah, V., Renaud, J.-P., Meinecke, R., Schlinck, N., Sitte, A., Popp, F., Zeeb, M., and Klebe, G. (2015) One Question, Multiple Answers: Biochemical and Biophysical Screening Methods Retrieve Deviating Fragment Hit Lists, ChemMedChem 10, 1511-1521.

2. Köster, H., Craan, T., Brass, S., Herhaus, C., Zentgraf, M., Neumann, L., Heine, A., and Klebe, G. (2011) A small nonrule of 3 compatible fragment library provides high hit rate of endothiapepsin crystal structures with various fragment chemotypes, J. Med. Chem. 54, 7784-7796.

3. Rühmann, E., Betz, M., Heine, A., and Klebe, G. (2015) Fragment Binding Can Be Either More Enthalpy-Driven or Entropy-Driven: Crystal Structures and Residual Hydration Patterns Suggest Why, Journal of Medicinal Chemistry 58, 6960-6971.

4. $\quad$ Collins, C. (2015) Diploma Thesis Universities of Marburg \& Halle.

5. Baum, B., Mohamed, M., Zayed, M., Gerlach, C., Heine, A., Hangauer, D., and Klebe, G. (2009) More than a simple lipophilic contact: a detailed thermodynamic analysis of nonbasic residues in the s1 pocket of thrombin, $J$ Mol Biol 390, 56-69.

6. Kuhnert, M., Koster, H., Bartholomäus, R., Park, A. Y., Shahim, A., Heine, A., Steuber, H., Klebe, G., and Diederich, W. E. (2015) Tracing binding modes in hit-to-lead optimization: chameleon-like poses of aspartic protease inhibitors, Angew. Chem. Int. Ed. Engl. 54, 2849-2853. 